\title{
A Proposal for a New Block Encryption Standard
}

\author{
Xuejia Lai James L. Massey \\ Institute for Signal and Information Processing \\ Swiss Federal Institute of Technology \\ CH-8092 Zürich, Switzerland
}

\section{Abstract}

A new secret-key block cipher is proposed as a candidate for a new encryption standard. In the proposed cipher, the plaintext and the ciphertext are 64 bit blocks, while the secret key is 128 bit long. The cipher is based on the design concept of "mixing operations from different algebraic groups". The cipher structure was chosen to provide confusion and diffusion and to facilitate both hardware and software implementations.

\section{Introduction}

A new secret-key block cipher is proposed herein as a candidate for a new encryption standard. In the proposed cipher, the plaintext and the ciphertext are 64 bit blocks, while the secret key is 128 bit long. The cipher is based on the design concept of "mixing operations from different algebraic groups". The required confusion is achieved by successively using three different group operations on pairs of 16-bit subblocks and the cipher structure was chosen to provide the necessary diffusion. The cipher is so constructed that the deciphering process is the same as the enciphering process once the decryption key subblocks have been computed from the encryption key subblocks. The cipher structure was chosen to facilitate both hardware and software implementations.

The cipher is described in Section 2. Section 3 considers the relation of the three chosen different operations to one another and the effect of their "mixing". The design principles for the cipher are discussed in Section 4. Section 5 discusses 
the implementation of the cipher in software as well as in hardware. A C-language program of the cipher is given in Appendix B together with examples that can be used to test the correctness of implementations.

\section{Description of the Proposed Cipher}

The computational graph of the encryption process is shown in Fig.1. The process consists of 8 similar rounds followed by an output transformation. The complete first round and the output transformation are depicted in Fig.1.

In the encryption process shown in Fig.1, three different group operations on pairs of 16-bit subblocks are used, namely,

- bit-by-bit exclusive-OR of two 16-bit subblocks, denoted as $\oplus$;

- addition of integers modulo $2^{16}$ where the subblock is treated as the usual radix-two representation of an integer, the resulting operation is denoted as $\boxplus$;

- multiplication of integers modulo $2^{16}+1$ where the subblock is treated as the usual radix-two representation of an integer except that the all-zero subblock is treated as representing $2^{16}$, and the resulting operation is denoted as $\odot$.

For example,

$$
(0, \ldots, 0) \odot(1,0, \ldots, 0)=(1,0, \ldots, 0,1)
$$

because

$$
2^{16} 2^{15} \bmod \left(2^{16}+1\right)=2^{15}+1 .
$$

The 64-bit plaintext block is partitioned into four 16-bit subblocks, the i-th of which is denoted as $X_{i}$ in Fig.1. The four plaintext subblocks are then transformed into four 16-bit ciphertext subblocks, $Y_{1}, Y_{2}, Y_{3}$ and $Y_{4}$, under the control of 52 key subblocks of 16 bits, where the six key subblocks used in the $r$-th $(r=1, \ldots, 8)$ round are denoted as $Z_{1}^{(r)}, . ., Z_{6}^{(r)}$ and where the four key subblocks used in the output transformation are denoted as $Z_{1}^{(9)}, Z_{2}^{(9)}, Z_{3}^{(9)}, Z_{4}^{(9)}$. 


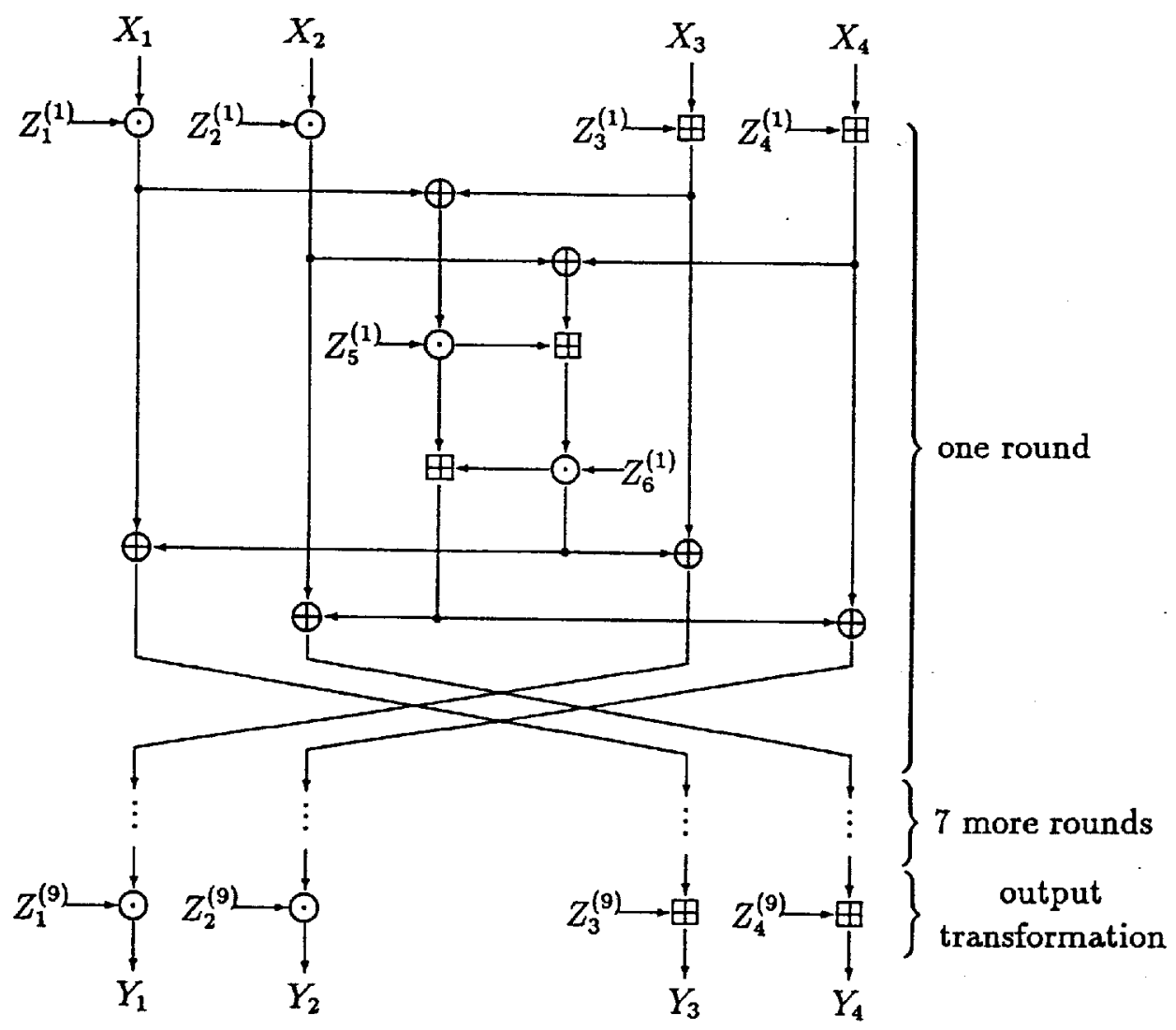

$X_{i}: 16$-bit plaintext subblock

$Y_{i}:$ 16-bit ciphertext subblock

$Z_{i}^{(r)}: 16$-bit key subblock

$\bigoplus$ : bit-by-bit exclusive-OR of 16-bit subblocks

$\boxplus$ : addition modulo $2^{16}$ of 16 -bit integers

$\odot$ : multiplication modulo $2^{16}+1$ of 16 -bit integers with the zero subblock corresponding to $2^{n}$

Figure 1: Computational graph for encryption 


\section{Decryption}

The computational graph of the decryption process is essentially the same as that of the encryption process, the only change being that the decryption key subblocks are computed from the encryption key subblocks as shown in the following table, where $Z^{-1}$ denotes the multiplicative inverse (modulo $2^{16}+1$ ) of $Z$, i.e., $Z \odot Z^{-1}=1$ and $-Z$ denotes the additive inverse (modulo $2^{16}$ ) of $Z$, i.e., $-Z \boxplus Z=0$.

Encryption key subblocks

Decryption key subblocks

\begin{tabular}{|c|c|}
\hline $\begin{array}{c}\text { 1-st } \\
\text { round }\end{array}$ & $\begin{array}{c}Z_{1}^{(1)} Z_{2}^{(1)} Z_{3}^{(1)} Z_{4}^{(1)} \\
Z_{5}^{(1)} Z_{6}^{(1)}\end{array}$ \\
\hline $\begin{array}{c}\text { 2-nd } \\
\text { round }\end{array}$ & $Z_{1}^{(2)} Z_{2}^{(2)} Z_{3}^{(2)} Z_{4}^{(2)}$ \\
\hline $\begin{array}{c}\text { 3-rd } \\
\text { round }\end{array}$ & $Z_{1}^{(3)} Z_{6}^{(3)} Z_{3}^{(3)} Z_{4}^{(3)}$ \\
\hline $\begin{array}{c}(3) \\
\text { 4-th }\end{array}$ & $Z_{1}^{(3)} Z_{2}^{(4)} Z_{3}^{(4)} Z_{4}^{(4)}$ \\
round & $Z_{5}^{(4)} Z_{6}^{(4)}$ \\
\hline $\begin{array}{c}\text { 5-th } \\
\text { round }\end{array}$ & $Z_{1}^{(5)} Z_{2}^{(5)} Z_{3}^{(5)} Z_{4}^{(5)}$ \\
\hline $\begin{array}{c}\text { 6-th } \\
\text { round }\end{array}$ & $Z_{1}^{(6)} Z_{6}^{(6)} Z_{3}^{(6)} Z_{4}^{(6)}$ \\
\hline $\begin{array}{c}\text { 7-th } \\
\text { round }\end{array}$ & $Z_{5}^{(6)} Z_{6}^{(6)} Z_{2}^{(7)} Z_{3}^{(7)} Z_{4}^{(7)}$ \\
\hline $\begin{array}{c}\text { 8-th } \\
\text { round }\end{array}$ & $Z_{1}^{(7)} Z_{6}^{(7)} Z_{2}^{(8)} Z_{3}^{(8)} Z_{4}^{(8)}$ \\
\hline output & $Z_{5}^{(8)} Z_{6}^{(8)}$ \\
\hline transform. & $Z_{1}^{(9)} Z_{2}^{(9)} Z_{3}^{(9)} Z_{4}^{(9)}$ \\
\hline
\end{tabular}

\begin{tabular}{|c|c|}
\hline $\begin{array}{c}1 \text {-st } \\
\text { round }\end{array}$ & $\begin{array}{c}Z_{1}^{(9)^{-1}} Z_{2}^{(9)^{-1}}-Z_{3}^{(9)}-Z_{4}^{(9)} \\
Z_{5}^{(8)} Z_{6}^{(8)}\end{array}$ \\
\hline $\begin{array}{l}2 \text {-nd } \\
\text { round }\end{array}$ & $\begin{array}{c}Z_{1}^{(8)^{-1}} Z_{2}^{(8)^{-1}}-Z_{3}^{(8)}-Z_{4}^{(8)} \\
Z_{5}^{(7)} Z_{6}^{(7)}\end{array}$ \\
\hline $\begin{array}{l}3 \text {-rd } \\
\text { round }\end{array}$ & $\begin{array}{c}Z_{1}^{(7)^{-1}} Z_{2}^{(7)^{-1}}-Z_{3}^{(7)}-Z_{4}^{(7)} \\
Z_{5}^{(6)} Z_{6}^{(6)}\end{array}$ \\
\hline $\begin{array}{l}4 \text {-th } \\
\text { round }\end{array}$ & $\begin{array}{c}Z_{1}^{(6)^{-1}} Z_{2}^{(6)}-Z_{3}^{(6)}-Z_{4}^{(6)} \\
Z_{5}^{(5)} Z_{6}^{(5)}\end{array}$ \\
\hline $\begin{array}{l}5 \text {-th } \\
\text { round }\end{array}$ & $\begin{array}{c}Z_{1}^{(5)^{-1}} Z_{2}^{(5)^{-1}}-Z_{3}^{(5)}-Z_{4}^{(5)} \\
Z_{5}^{(4)} Z_{6}^{(4)}\end{array}$ \\
\hline $\begin{array}{l}6 \text {-th } \\
\text { round }\end{array}$ & $\begin{array}{c}Z_{1}^{(4)^{-1}} Z_{2}^{(4)^{-1}}-Z_{3}^{(4)}-Z_{4}^{(4)} \\
Z_{5}^{(3)} Z_{6}^{(3)}\end{array}$ \\
\hline $\begin{array}{l}7 \text {-th } \\
\text { round }\end{array}$ & $\begin{array}{c}Z_{1}^{(3)^{-1}} Z_{2}^{(3)^{-1}}-Z_{3}^{(3)}-Z_{4}^{(3)} \\
Z_{5}^{(2)} Z_{6}^{(2)}\end{array}$ \\
\hline $\begin{array}{l}8 \text {-th } \\
\text { round }\end{array}$ & $\begin{array}{c}Z_{1}^{(2)^{-1}} Z_{2}^{(2)^{-1}}-Z_{3}^{(2)}-Z_{4}^{(2)} \\
Z_{5}^{(1)} Z_{6}^{(1)}\end{array}$ \\
\hline $\begin{array}{c}\text { output } \\
\text { transform. }\end{array}$ & $Z_{1}^{(1)^{-1}} Z_{2}^{(1)^{-1}}-Z_{3}^{(1)}-Z_{4}^{(1)}$ \\
\hline
\end{tabular}

\section{The key schedule}

The 52 key subblocks used in the encryption process are generated from the 128 bit user-selected key as follows:

The 128 bit user-selected key is partitioned into 8 subblocks that are directly used as the first eight key subblocks, where the ordering of key subblocks is as follows: $Z_{1}^{(1)}, Z_{2}^{(1)}, \ldots, Z_{6}^{(1)}, Z_{1}^{(2)}, \ldots, Z_{6}^{(2)}, \ldots, Z_{1}^{(8)}, . ., Z_{6}^{(8)}, Z_{1}^{(9)}, Z_{2}^{(9)}, Z_{3}^{(9)}, Z_{4}^{(9)}$.

The 128 bit user-selected key is then cyclic shifted to the left by 25 positions, after which the resulting 128 bit block is again partitioned into eight subblocks that are 
taken as the next eight key subblocks. The obtained 128 bit block is cyclic shifted again to the left by 25 positions to produce the next eight key subblocks, and this procedure is repeated until all 52 key subblocks have been generated.

\section{Group Operations and Their Interaction}

The cipher is based on the design concept of "mixing operations from different algebraic groups having the same number of elements". Group operations were chosen because the statistical relation of random variables $X, Y, Z$ related by a group operation as $Y=X * Z$ has the desired "perfect secrecy" property, i.e., if one of the random variables is chosen equally likely to be any group element, then the other two random variables are statistically independent. The interaction of different group operations contributes to the "confusion" required for a secure cipher. In this section, the interaction of different operations will be considered in terms of isotopism of quasigroups and in terms of polynomial expressions.

\section{The three operations as quasigroup operations}

Def. Let $S$ be a set and let $*$ denote an operation from pairs $(a, b)$ of elements of $\mathrm{S}$ to an element $a * b$ of $\mathrm{S}$. Then $(S, *)$ is said to be an quasigroup if, for any $a, b \in S$, the equations $a * x=b$ and $y * a=b$ both have exactly one solution in S. A group is a quasigroup in which the operation is associative, i.c., for which $a *(b * c)=(a * b) * c$ for all $a, b$ and $c$ in S. Quasigroups $\left(S_{1}, *_{1}\right),\left(S_{2}, *_{2}\right)$ are said to be isotopic if there are bijective mappings $\theta, \phi, \psi: S_{1} \rightarrow S_{2}$, such that, for all $x, y \in S_{1}, \theta(x) *_{2} \phi(y)=\psi\left(x *_{1} y\right)$. Such a triple $(\theta, \phi, \psi)$ is then called an isotopism of $\left(S_{1}, *_{1}\right)$ upon $\left(S_{2}, *_{2}\right)$. Two groups are said to be isomorphic if they are isotopic as quasigroups for which the isotopism is $(\theta, \theta, \theta)$.

It can be shown that two groups are isomorphic if and only if they are isotopic [3].

Let $n$ be one of the integers $1,2,4,8$ or 16 so that the integer $2^{n}+1$ is a prime, and let $Z_{2^{n}}$ denote the ring of integers modulo $2^{n}$. Let $\left(Z_{2^{n}+1}^{*} \cdot\right)$ denote the multiplicative group of the non-zero elements of the field $Z_{2^{n}+1}$, let $\left(Z_{2 n},+\right)$ denote the additive group of the ring $Z_{2^{n}}$, and let $\left(F_{2}^{n}, \oplus\right)$ denote the group of n-tuples over $F_{2}$ under the bitwise exclusive-or operation. Then the following theorem states some of the "incompatibility" properties of these groups.

Theorem 1 For $n \in\{1,2,4,8,16\}$ :

1) Quasigroups $\left(F_{2}^{n}, \oplus\right)$ and $\left(Z_{2^{n}},+\right)$ are not isotopic for $n \geq 2$.

2) Quasigroups $\left(Z_{2^{n}+1}^{*}, \cdot\right)$ and $\left(F_{2}^{n}, \oplus\right)$ are not isotopic for $n \geq 2$.

3) $(\theta, \phi, \psi)$ is an isotopism of $\left(Z_{2^{n}+1}^{*}, \cdot\right)$ upon $\left(Z_{2^{n}},+\right)$ if and only if there exist constants $c_{1}, c_{2} \in Z_{2^{n}}$ and a primitive element $a$ of the field $Z_{2^{n}+1}^{*}$ such that, for all $x$ in $Z_{2^{n}}$,

$$
\theta(x)-c_{1}=\phi(x)-c_{2}=\psi(x)-\left(c_{1}+c_{2}\right)=\log _{a}(x),
$$


i.e., any isotopism between these groups is essentially the logarithm. Moreover, if $(\theta, \phi, \psi)$ is an isotopism, none of these maps will be the "mixing mapping" $m$ from $Z_{2^{n}+1}^{*}$ to $Z_{2^{n}}$ defined by $m(i)=i$, for $i \neq 2^{n}$ and $m\left(2^{n}\right)=0$ when $n \geq 2$.

\section{Proof.}

1) For $n \geq 2$, the groups $\left(F_{2}^{n}, \oplus\right)$ and $\left(Z_{2^{n}},+\right)$ are not isomorphic because $\left(Z_{2^{n}},+\right)$ is a cyclic group while $\left(F_{2}^{n}, \oplus\right)$ is not. Thus, they are not isotopic as quasigroups.

2) $\left(Z_{2^{n}+1}^{*}, \cdot\right)$ and $\left(Z_{2^{n}},+\right)$ are isomorphic groups for $n=1,2,4,8,16$ because both groups are cyclic. Thus, $\left(Z_{2^{n}+1}^{*}, \cdot\right)$ is isotopic to $\left(F_{2}^{n}, \oplus\right)$ if and only if $\left(Z_{2^{n}},+\right)$ is isotopic to $\left(F_{2}^{n}, \oplus\right)$.

3) If (1) holds, then for any $x, y$ in $Z_{2^{n}+1}$,

$$
\psi(x \cdot y)=\log _{a}(x \cdot y)+c_{1}+c_{2}=\log _{a}(x)+\log _{a}(y)+c_{1}+c_{2}=\theta(x)+\phi(y) .
$$

Now suppose that $(\theta, \phi, \psi)$ is an isotopism. Then for all $x, y \in Z_{2^{n}+1}^{*}, \theta(x)+\phi(y)=$ $\psi(x \cdot y)$. Let $\theta_{1}(x)=\theta(x)-\theta(1), \phi_{1}(x)=\phi(x)-\phi(1)$ and $\psi_{1}(x)=\psi(x)-\psi(1)$, then $\left(\theta_{1}, \phi_{1}, \psi_{1}\right)$ is also an isotopism and $\psi_{1}(1)=0_{1}(1)=\phi_{1}(1)=0$. In the equation $\theta_{1}(x)+\phi_{1}(y)=\psi_{1}(x \cdot y)$, setting first $x$ and then $y$ to 1 results in $\theta_{1}(y)=\phi_{1}(y)=\psi_{1}(y)$ so that $\psi_{1}(x \cdot y)=\psi_{1}(x)+\psi_{1}(y)$. Let $a$ be the element of $Z_{2^{n}+1}^{*}$ such that $\psi_{1}(a)=1$, then $\psi_{1}\left(a^{i}\right)=i$ for $i=1,2, . .2^{n}-1$ and $\psi_{1}\left(a^{2^{n}}\right)=0$. This implies that $a$ is a primitive element of $Z_{2^{n}+1}$. Thus, for each $x \in Z_{2^{n}+1}^{*}$, there exists a $t \in Z_{2^{n}}$ such that,

$$
\psi_{1}(x)=\psi_{1}\left(a^{t}\right)=t=t \log _{a}(a)=\log _{a}\left(a^{t}\right)=\log _{a}(x) .
$$

Letting $c_{1}=O(1), c_{2}=\phi(1)$, we arrive at equation (1).

Finally, suppose that the mixing mapping $m$ is an isotopism. Then $m(x)=$ $\log _{a}(x)+c$ implies $1=m(1)=\log _{a}(1)+c=c, 2=m(2)=\log _{a}(2)+1$, which implies that $a=2$, and $0=m\left(2^{n}\right)=\log _{2}\left(2^{n}\right)+1=n+1$, which implies that $\mathrm{n}=1$.

\section{Polynomial expressions for multiplication and addition}

Under the mixing mapping $m$, multiplication modulo $2^{n}+1$, which is a bilinear function over the field $Z_{2^{n}+1}$, is a two variable function over the ring $Z_{2^{n}}$, which we denote by $x \odot y$. Similarly, under the inverse mixing mapping $m^{-1}$, addition modulo $2^{n}$, which is an affine function in each argument over the ring $Z_{2^{n}}$, is a two variable function over the field $Z_{2^{n}+1}$, which we denote by $F(X, Y)$. Here and hereafter in this section, we denote arguments with lower-case letters when we consider them to be elements of $Z_{2^{n}}$ and with upper-case letters when we consider them to be elements of $Z_{2^{n}+1}$. For example, when $n=1$, we have

$x+y \bmod 2 \longleftrightarrow F(X, Y)=2 X Y \bmod 3$,

$X Y \bmod 3 \longleftrightarrow x \odot y=x+y+1 \bmod 2$. 
Theorem 2 For $n \in\{2,4,8,16\}$ :

1. For any fixed $X \neq 2^{n}$ (i.e., $\left.x \neq 0\right)$, the function $F(X, Y)$, corresponding to addition $x+y \bmod 2^{n}$ in $Z_{2^{n}}$, is a polynomial in $Y$ over $Z_{2^{n}+1}$ with degree $2^{n}-1$. Similarly, for any fixed $Y \neq 2^{n}, F(X, Y)$ is a polynomial in $X$ over $Z_{2^{n}+1}$ with degree $2^{n}-1$. 2. For any fixed $x \neq 0,1$ (i.e., $X \neq 2^{n}, 1$ ), the function $x \odot y$, corresponding to multiplication $X Y \bmod 2^{n}+1$ in $Z_{2^{n}+1}$ cannot be written as a polynomial in $y$ over $Z_{2^{n}}$. Similarly, for any fixed $y \neq 0,1, x \odot y$ is not a polynomial in $x$ over $Z_{2^{n}}$.

\section{Example 1}

For $n=2$, in $Z_{5}$, the function $F(X, Y)$ corresponding to $x+y \bmod 4$ is

$$
F(X, Y)=3\left(X^{3} Y^{2}+X^{2} Y^{3}\right)+3\left(X^{3} Y+X Y^{3}\right)+2 X^{2} Y^{2}+4\left(X^{2} Y+X Y^{2}\right) .
$$

\section{Proof.}

1. In any finite field $G F(q)$, we have

$$
\prod_{j \neq i, \alpha_{j} \neq 0}\left(x-\alpha_{j}\right)\left(-\alpha_{i}\right)= \begin{cases}\prod_{j(j \neq i), \alpha_{j} \neq 0}\left(\alpha_{i}-\alpha_{j}\right)\left(-\alpha_{i}\right)=-\prod_{\alpha_{i} \neq 0} \alpha_{i}=1 & x=\alpha_{i} \\ 0 & x \neq \alpha_{i}, 0 .\end{cases}
$$

Thus, every function $f(x)$ from the set $G F(q)-\{0\}$ to $G F(q)-\{0\}$ can be written as a polynomial over GF(q) of degrce at most $q-2$ as follows:

$$
f(x)=\sum_{\alpha_{i} \in G F(q)-\{0\}} f\left(\alpha_{i}\right) \prod_{\alpha_{j} \neq \alpha_{i}, 0}\left(x-\alpha_{j}\right)\left(-\alpha_{i}\right) .
$$

The function $F(A, X)$ corresponding to $a+x \bmod 2^{n}$ is a function from $G F\left(2^{n}+1\right)-\{0\}$ to $G F\left(2^{n}+1\right)-\{0\}$ in X. According to (2), this function can be written as

$$
\begin{aligned}
F(A, X) & = \begin{cases}A+X & 1 \leq X \leq 2^{n}-A \\
A+X+1 & 2^{n}-A<X \leq 2^{n}\end{cases} \\
& =\sum_{I=1}^{2^{n}-A}(A+I) \prod_{\substack{J \neq I \\
1 \leq J \leq 2^{n}}}(X-J)(-I)+\sum_{I=2^{n}-A+1}^{2^{n}}(A+I+1) \prod_{\substack{J \neq I \\
1 \leq J \leq 2^{n}}}(X-J)(-I) \\
& =\sum_{I=1}^{2^{n}}(A+I) \prod_{\substack{J \neq I \\
1 \leq J \leq 2^{n}}}(X-J)(-I)+\sum_{I=2^{n}-A+1}^{2^{n}} \prod_{\substack{J \neq I \\
1 \leq J \leq 2^{n}}}(X-J)(-I)
\end{aligned}
$$

The coefficient of $X^{2^{n}-1}$ in $F(A, X)$ is

$$
\begin{aligned}
& \sum_{I=1}^{2^{n}}(A+I)(-I)+\sum_{I=2^{n}-A+1}^{2^{n}}(-I)=-A \sum_{I=1}^{2^{n}} I-\sum_{I=1}^{2^{n}} I^{2}+\sum_{I=-A}^{-1}(-I) \\
= & \sum_{I=1}^{A} I=\frac{A(A+1)}{2},
\end{aligned}
$$

which is zero if and only if $A=0$ or $A=-1=2^{n}$, which cases are excluded by the assumption. Thus, $\operatorname{deg} F(X, A)=2^{n}-1$.

2. We show first the following lemma: 
Lemma 1 If $f(x)$ is a polynomial over $Z_{2^{n}}$, then for all $x \in Z_{2^{n}}$,

$$
f(2 x) \bmod 2=f(0) \bmod 2 .
$$

Proof. Let $f(x)=a_{k} x^{k}+a_{k-1} x^{k-1}+\cdots+a_{1} x+a_{0}$.

Then for all $x \in Z_{2^{n}}$,

$$
f(2 x)=a_{k}(2 x)^{k}+a_{k-1}(2 x)^{k-1}+\cdots+a_{1} 2 x+a_{0} .
$$

Taking both sides of this equation modulo $2^{n}$, we have $f(2 x)=2 e+a_{0} \bmod 2^{n}$, where $e$ is an element in $Z_{2^{n}}$. Thus, $f(2 x) \bmod 2=a_{0} \bmod 2=f(0) \bmod 2$.

Now let $n>1$, then for every integer $a, 1<a<2^{n}$, there exists an integer $x_{0} \in\left\{1,2, \ldots, 2^{n}\right\}$ such that $2^{n}+1<2 a x_{0}<2\left(2^{n}+1\right)$ and $0 \leq 2 a\left(x_{0}-1\right)<2^{n}+1$. The first inequality is equivalent to that $0<2 a x_{0}-\left(2^{n}+1\right)<2^{n}+1$ and $2 a x_{0}-\left(2^{n}+1\right)$ is an odd integer. Hence the function $f_{a}(x)=a \odot x$ corresponding to $A X \bmod \left(2^{n}+1\right)$ satisfies

$$
f_{a}\left(2 x_{0}\right) \bmod 2=\left(2 a x_{0}-\left(2^{n}+1\right)\right) \bmod 2=1 .
$$

On the other hand, the inequality $0 \leq 2 a\left(x_{0}-1\right)<2^{n}+1$ implies that $2 a\left(x_{0}-1\right)$ is an even integer in $\left\{0,1, \ldots, 2^{n}\right\}$ so that

$$
f_{a}\left(2\left(x_{0}-1\right)\right) \bmod 2=2 a\left(x_{0}-1\right) \bmod 2=0 .
$$

By the Lemma, $f_{a}(x)$ is not a polynomial over $Z_{2^{n}}$.

\section{Design Principles for the Proposed Cipher}

\section{Confusion}

Confusion (see $[1,2]$ ) means that the ciphertext depends on the plaintext and key in a complicated and involved way.

The confusion is achieved by mixing three different group operations. In the computational graph of the encryption process, the three different group operations are so arranged that the output of an operation of one type is never used as the input to an operation of the same type.

The three operations are incompatible in the sense that:

1. No pair of the 3 operations satisfies a distributive law. For example,

$$
a \boxplus(b \odot c) \neq(a \boxplus b) \odot(a \boxplus c) .
$$

2. No pair of the 3 operations satisfies an associative law. For example,

$$
a \boxplus(b \oplus c) \neq(a \boxplus b) \oplus c
$$


3. The 3 operations are combined by the mixing mapping $m$, which inhibits isotopisms as was shown in Theorem 1. Thus, using any bijections on the operands, it is impossible to realize any one of the three operations by another operation.

4. Under the mixing mapping, multiplication modulo $2^{16}+1$, which is a bilinear function over $Z_{2^{16}+1}$, corresponds a non-polynomial function over $Z_{2^{16}}$; Under the inverse mixing mapping, addition modulo $2^{16}$, which is an affine function in each argument over $Z_{2^{16}}$, corresponds a two variable polynomial of degree $2^{16}-1$ in each variable over $Z_{2^{16}+1}$.

\section{Diffusion}

The diffusion requirement on a cipher is that each plaintext bit should influence every ciphertext bit and each key bit should influence every ciphertext bit(see $[1,2])$.

For the proposed cipher, a check by computer has shown that the diffusion requirement is satisfied after the first round, i.e., each output bit of the first round depends on every bit of the plaintext and on every bit of the key used for that round.

Diffusion is provided by the transformation called the multiplication-addition (MA) structure. The computational graph of the MA structure is shown in Fig.2. The MA structure transforms two 16 bit subblocks into two 16 bit subblocks controlled by two 16 bit key subblocks. This structure has the following properties:

- for any choice of the key subblocks $Z_{5}$ and $Z_{6}, M A\left(\cdot, \cdot, Z_{5}, Z_{6}\right)$ is an invertible transformation; for any choice of $U_{1}$ and $U_{2}, M A\left(U_{1}, U_{2}, \cdot, \cdot\right)$ is also an invertible transformation;

- this structure has a "complete diffusion" effect in the sense that each output subblock depends on every input subblock, and

- this structure uses the least number of operations (four) required to achieve such complete diffusion (see Appendix A for the proof).

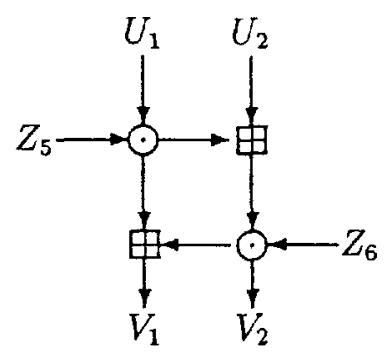

Figure 2: Computational graph of the MA structure 


\section{Similarity of encryption and decryption}

The similarity of encryption and decryption means that decryption is essentially the same process as encryption, the only difference being that different key subblocks are used. This similarity results from

- using the output transformation in the encryption process so that the effect of $\left(Z_{1}, Z_{2}, Z_{3}, Z_{4}\right)$ can be cancelled by using inverse key subblocks $\left(Z_{1}^{-1}, Z_{2}^{-1},-Z_{3}\right.$, $\left.-Z_{4}\right)$ in the decryption process,

and from

- using an involution (i.e., a self-inverse function) with a 64 bit input and a 64 bit output controlled by a 32 bit key within the cipher. The involution used in the cipher is shown in Fig.3. The self-inverse property is a consequence of the fact that the exclusive-OR of $\left(S_{1}, S_{2}\right)$ and $\left(S_{3}, S_{4}\right)$ is equal to the exclusive-OR of $\left(T_{1}, T_{2}\right)$ and $\left(T_{3}, T_{4}\right)$; Thus, the input to the MA structure in Fig.3 is unchanged when $S_{1}, S_{2}, S_{3}$ and $S_{4}$ are replaced by $T_{1}, T_{2}, T_{3}$ and $T_{4}$. Thus, if $T_{1}, T_{2}, T_{3}$ and $T_{4}$ are the inputs to the involution, the left half of the output is

$$
\begin{aligned}
& \left(T_{3}, T_{4}\right) \oplus M A\left(\left(T_{1}, T_{2}\right) \oplus\left(T_{3}, T_{4}\right), Z_{5}, Z_{6}\right) \\
= & \left(S_{1}, S_{2}\right) \oplus M A\left(\left(S_{1}, S_{2}\right) \oplus\left(S_{3}, S_{4}\right), Z_{5}, Z_{6}\right) \oplus M A\left(\left(S_{1}, S_{2}\right) \oplus\left(S_{3}, S_{4}\right), Z_{5}, Z_{6}\right) \\
= & \left(S_{1}, S_{2}\right) .
\end{aligned}
$$

Similarly, the right half of the output is $\left(S_{3}, S_{4}\right)$.

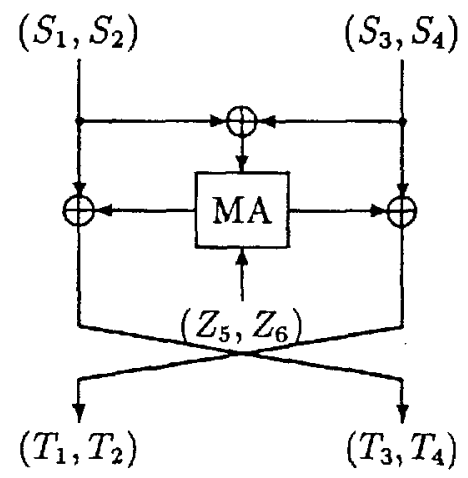

Figure 3: Computational graph of the involution

Perfect secrecy for a "one-time" key

Perfect secrecy in the sense of Shannon is obtained in each round of encryption if a "one-time" key is used. In fact, such perfect secrecy is achieved at the input transformation in the first round because each operation is a group operation. In addition, 
for every choice of $\left(p_{1}, p_{2}, p_{3}, p_{4}\right)$ and of $\left(q_{1}, q_{2}, q_{3}, q_{4}\right)$ in $F_{2}^{64}$, there are exactly $2^{32}$ different choices of the key subblocks $\left(Z_{1}, . ., Z_{6}\right)$ such that the first round of the cipher transforms $\left(p_{1}, p_{2}, p_{3}, p_{4}\right)$ into $\left(q_{1}, q_{2}, q_{3}, q_{4}\right)$.

\section{Implementations of the Cipher}

The cipher structure was chosen to facilitate both hardware and software implementations. In the encryption process, a regular modular structure was chosen so that the cipher can be easily implemented in hardware. A VLSI implementation of the cipher is being carried out at the Integrated Systems Laboratory of the ETH, Zürich. The estimated data rate of this chip varies from 45 Mbits per second to $115 \mathrm{Mbits}$ per second, depending on the complexity of the architecture chosen.

The cipher can also be easily implemented in software because only operations on pairs of 16-bit subblocks are used in the encryption process.

The most difficult part in the implementation, multiplication modulo $\left(2^{16}+1\right)$, can be implemented in the way suggested by the following lemma.

Lemma 2 Let $a, b$ be two $n$-bit non-zero integers in $Z_{2^{n}+1}$, then

$a b \bmod \left(2^{n}+1\right)= \begin{cases}\left(a b \bmod 2^{n}\right)-\left(a b \operatorname{div} 2^{n}\right) & \text { if }\left(a b \bmod 2^{n}\right) \geq\left(a b \operatorname{div} 2^{n}\right) \\ \left(a b \bmod 2^{n}\right)-\left(a b \operatorname{div} 2^{n}\right)+2^{n}+1 & \text { if }\left(a b \bmod 2^{n}\right)<\left(a b \operatorname{div} 2^{n}\right)\end{cases}$

where $\left(a b \operatorname{div} 2^{n}\right)$ denotes the quotient when $a b$ is divided by $2^{n}$.

Note that $\left(a b \bmod 2^{n}\right)$ corresponds to the $\mathrm{n}$ least significant bits of $a b$, and $\left(a b \operatorname{div} 2^{n}\right)$ is just the right-shift of $a b$ by $\mathrm{n}$ bits. Note also that $\left(a b \bmod 2^{n}\right)=$ $\left(a b \operatorname{div} 2^{n}\right)$ implies that $a b \bmod \left(2^{n}+1\right)=0$ and hence cannot occur when $2^{n}+1$ is a prime.

Proof. For any non-zero $a$ and $b$ in $Z_{2^{n}+1}$, there exist unique integers $q$ and $r$ such that

$$
a b=q\left(2^{n}+1\right)+r, \quad 0 \leq r<2^{n}+1,0 \leq q<2^{n} .
$$

Moreover, $q+r<2^{n+1}$. Note that $r=a b \bmod \left(2^{n}+1\right)$. We have

$$
\left(a b \operatorname{div} 2^{n}\right)= \begin{cases}q & \text { if } q+r<2^{n} \\ q+1 & \text { if } q+r \geq 2^{n}\end{cases}
$$

and

$$
\left(a b \bmod 2^{n}\right)= \begin{cases}q+r & \text { if } q+r<2^{n} \\ q+r-2^{n} & \text { if } q+r \geq 2^{n}\end{cases}
$$

Thus

$$
r= \begin{cases}\left(a b \bmod 2^{n}\right)-\left(a b \operatorname{div} 2^{n}\right) & \text { if } q+r<2^{n} \\ \left(a b \bmod 2^{n}\right)-\left(a b \operatorname{div} 2^{n}\right)+2^{n}+1 & \text { if } q+r \geq 2^{n}\end{cases}
$$


But $q+r<2^{n}$ if and only if $\left(a b \bmod 2^{n}\right) \geq\left(a b \operatorname{div} 2^{n}\right)$. This proves the Lemma.

A C-program of the cipher together with some examples for checking the correctness of the implementation are given in Appendix B.

\section{Conclusion}

The cipher described above is proposed as a possible candidate for a new encryption standard. The cipher is based on the design concept of "mixing 3 different group operations" to achieve the required confusion and diffusion. Confusion is achieved by arranging the operations in a way that no pair of successive operations are of the same type and by the fact that operations of different types are incompatible. The structure of the cipher is so chosen that diffusion can be achieved using a small number of operations.

Enciphering and deciphering are essentially the same process, but with different key subblocks. Because of the use of 16-bit operations and a regular modular structure, the cipher can be implemented efficiently in both hardware and software. In particular, bit-level permutations are avoided in the encryption process because such permutations are difficult to implement in software.

In all of the statistical testings conducted up to now, we have not found any significant difference between the permutation of $F_{2}^{64}$ determined by the cipher with a randomly chosen key and a permutation equiprobably chosen from all possible permutations of $F_{2}^{64}$.

The security of the proposed cipher needs further intensive investigation. The authors hereby invite interested parties to attack this proposed cipher and will be grateful to receive the results (positive or negative) of any such attacks.

\section{References}

[1] C. E. Shannon, "Communication Theory of Secrecy Systems", B.S.T.J., Vol. 28, pp.656-715, Oct. 1949.

[2] J. L. Massey, "An Introduction to Contemporary Cryptology", Proc. IEEE, Vol. 76, No. 5, pp. 533-549, May 1988.

[3] J.Dénes, A.D.Keedwell, Latin squares and their applications, Akadémiai Kiadó, Budapest 1974. 


\section{Appendix A: Number of Operations Required for Diffusion}

An operation is a mapping from two variables to one variable. A computational graph is a directed graph in which the vertices are operations, the edges entering a vertex are the inputs to the operation, the edges leaving a vertex are the outputs of the operation, the edges entering no vertex are the graph outputs, and the edges leaving no vertex are the graph inputs. An algorithm to compute a function determines a computational graph where the graph inputs are the inputs of the algorithm and the graph outputs are the outputs of the algorithm.

Consider a function having the form

$$
\left(Y_{1}, Y_{2}\right)=E\left(X_{1}, X_{2}, Z_{1}, Z_{2}\right), \quad X_{i}, Y_{i} \in F_{2}^{m}, \quad X_{i} \in F_{2}^{k}
$$

such that, for every choice of $\left(Z_{1}, Z_{2}\right), E\left(\cdot, \cdot, Z_{1}, Z_{2}\right)$ is invertible. Such a function will be called a cipher function. A cipher function is said to have complete diffusion if each of its output variable depends non-idly on every input variable.

Theorem 3 If a cipher function of the form (3) has complete diffusion, then the computational graph of any algorithm that computes the function contains at least 4 operations.

Proof. Let $Y_{1}=E_{1}\left(X_{1}, X_{2}, Z_{1}, Z_{2}\right)$, and $Y_{2}=E_{2}\left(X_{1}, X_{2}, Z_{1}, Z_{2}\right)$. Then if $E_{1}$ has complete diffusion, it contains at least 3 operations because there are four input variables. Suppose $E_{1}$ contains 3 operations. The invertibility of the cipher function implies that $E_{2} \neq E_{1}$ and complete diffusion requires that $E_{2}$ not equal any intermediate result that appears in $E_{1}$. Thus, at least one operation not appearing in $E_{1}$ is required in $E_{2}$. This proves the theorem.

It is easy to check that the MA structure shown in Fig.2, which has four operations, is a cipher function with complete diffusion, i.e., that each of the output variables depends non-idly on all four input variables.

\section{Appendix B: C-program of the Cipher and Sample Data}

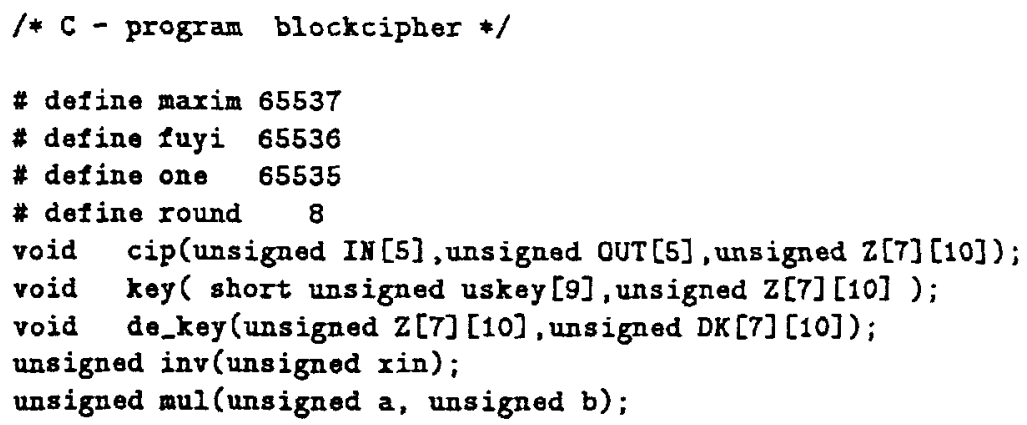




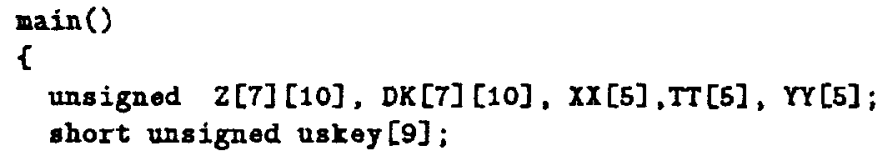

\}

\section{/* encryption algorithm */}

void cip(unsigned IX [5], unsigned OUT[5], unsigned $z[7][10]$ )

t

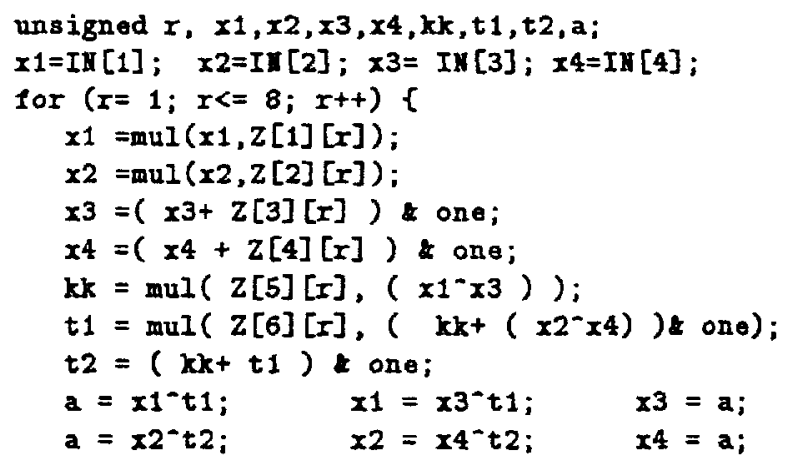

/* the multiplication */ unsigned mul (unsigned $a$, unsigned b) \{ 


\begin{tabular}{|c|c|c|c|c|c|c|}
\hline & {$[1][x]$} & $z[2][r] 2$ & $z[3][r]$ & $2[4] L$ & ZL! & 2 Lo. \\
\hline 1-st round & 1 & 2 & 3 & 4 & 5 & 6 \\
\hline 2-nd round & 7 & 8 & 1024 & 1536 & 2048 & 2560 \\
\hline 3-rd round & 3072 & 3584 & 4096 & 512 & 16 & 20 \\
\hline 4-th round & 24 & 28 & 32 & 4 & 8 & 12 \\
\hline 5-th round & 10240 & 12288 & 14336 & 16384 & 2048 & 4096 \\
\hline 6-th round & 6144 & 8192 & 112 & 128 & 16 & 32 \\
\hline 7 -th round & 48 & 64 & 80 & 96 & 0 & 8192 \\
\hline 8-th round & 16384 & 24576 & 32768 & 40960 & 49152,5 & 57345 \\
\hline output transf & 128 & 192 & 256 & 320 & - & -- \\
\hline decryption key s & subbloc & DKC & {$[i][I]$} & & & \\
\hline 1-8t round & 65025 & 43350 & 652806 & 65216 & $49152 \quad 5$ & 57345 \\
\hline 2-nd round & 65533 & 21843 & 32768 & 24576 & 0 & 8192 \\
\hline 3-rd round & 42326 & 64513 & 65456 & 65440 & 16 & 32 \\
\hline 4-th round & 21835 & 65529 & 65424 & 65408 & 2048 & 4096 \\
\hline 5-th round & 13101 & 43686 & 51200 & 49152 & 8 & 12 \\
\hline 6-th round & 19115 & 53834 & 65504 & 65532 & 16 & 20 \\
\hline 7-th round & 43670 & 28069 & 61440 & 65024 & 2048 & 2560 \\
\hline 8-th round & 18725 & 57345 & 64512 & 64000 & 5 & 6 \\
\hline output transf & 1 & 32769 & 65533 & 65532 & -- & -- \\
\hline plaintext $X$ & $x x$ & & 0 & 1 & 2 & 3 \\
\hline after 1-st roun & & 177 & 202 & 180 & 207 & \\
\hline after 2-nd roun & & 5054 & 10696 & 5085 & $5 \quad 10583$ & \\
\hline after 3-rd roun & & 42790 & 64040 & $0 \quad 25583$ & 315559 & \\
\hline after $4-$ th roun & & 16281 & 58571 & 61463 & $3 \quad 33861$ & \\
\hline after $5-$ th roun & & 62321 & 51187 & 1399 & 59053 & \\
\hline after 6-th roun & & 37668 & 1126 & 6125 & 542057 & \\
\hline after 7 -th roun & & 49700 & 61227 & $7 \quad 19644$ & $4 \quad 21245$ & \\
\hline after 8-th roun & & 2688 & 12695 & 2372 & 1339 & \\
\hline$Y Y=\operatorname{cip}(x x$, & $, z)$ & 16379 & $9 \quad 12571$ & 2628 & 1659 & \\
\hline$T T=\operatorname{cip}(Y Y, D K)$ & & & 0 & 1 & 2 & 3 \\
\hline
\end{tabular}

